\title{
Capital social y redes sociales: analisis del Tercer Sector en contextos rurales
}

\section{Isabel Saz-Gil, Patricia Almaguer-Kalixto y Juan David Gómez-Quintero}

\section{RESUMEN}

El tercer sector de acción social (TSAS) es uno de los pilares del sistema de bienestar particularmente estratégico en zonas rurales. Tomando el estudio de caso como base, este trabajo analiza el TSAS de Teruel, una provincia de Aragón (España) con población mayoritariamente rural, dispersa, y escasa. Presentamos evidencia cuantitativa y cualitativa de las redes sociales existentes y las relaciones entre las organizaciones e instituciones relevantes para el sistema de bienestar. Las conclusiones abordan una sensación generalizada de pertenencia al sector y territorio, que se relaciona con una fuerte identificación con el espacio local. En este caso, el capital social prioriza la fortaleza de los vínculos intrínsecos dentro de las redes intra-territoriales, sobre las extrínsecas o extraterritoriales. Sin embargo, se percibe que se necesitan nuevas estrategias y capitales para reforzar la conexión con las redes externas para favorecer la sostenibilidad del sector.

PALABRAS CLAVE: Tercer sector de acción social, capital social, redes, confianza, reciprocidad. CLAVES ECONLIT: L31, M19, 018, Z13.

Cómo citar este artículo / How to cite this article: SAZ-GIL, I., ALMAGUER-KALIXTO, P. \& GÓMEZQUINTERO, J.D. (2016): "Capital social y redes sociales: análisis del Tercer Sector en contextos rurales", CIRIEC-España, Revista de Economía Pública, Social y Cooperativa, 86, 123-154.

Correspondencia: Isabel Saz-Gil, Patricia Almaguer-Kalixto y Juan David Gómez-Quintero. E-mail de contacto: sazgil@unizar.es 


\section{EXPANDED ABSTRACT}

\section{Social capital and networks: analysis of the Third Sector in rural contexts}

\section{Background}

The third sector of social action is one of the mainstays of the welfare system. As such it has among its functions the ability to detect social requirements that have not been met and to enhance institutional solutions while encouraging social participation. According to Peraldi \& Rombaldi (2009) it is essential to integrate social organizations in weakened rural areas. But advances in such processes require that the social utility produced by these organizations is socially recognised. We argue that it is also important that the sector recognises its own network of relationships by identifying the sector strengths and weaknesses.

This paper considers the third sector of social action in Teruel, a province of Aragón in Spain. Our research analyses (a) the configuration of different dimensions of social capital, (b) how social capital is expressed through third sector of social action networks and (c) how the sector organises addressing challenges in primarily rural contexts. The research results are expected to enable a diagnosis of the sector in the province of Teruel, contributing to strengthening this sector.

\section{Methodology}

We chose the case study as the basis of the methodological design. Considering Flybjerg (2004) classification, we address Teruel an extreme case study due to its particular features: a combination of a low population rate, a high rate of aging and low capacity for population renewal due to migration trends. In addition the population is geographically dispersed across small communities in 236 municipalities with only two cities of more than 10,000 people. The combination of population and territory present a sparsely-populated province with one of the lowest population density rates in Spain at 941 inhabitants per square kilometer. Teruel is the province with the highest average population age at 45.1 years (Mur, 1998). To this scenario we need to add the government's reduction' in public spending on social services. We argue that third-sector organizations must overcome important challenges to accomplish their social intervention objectives, as the sustainability of social services in these areas of combined depopulation and aging is relevant and urgent.

The study relies on a mixed-method approach that integrates quantitative and qualitative data collection instruments. Following Li's (2015) observations on methods and strategies for the study of 
social capital, the first part of our design' includes a survey with 58 questions to gather information on variables related to the existing capital in the sector: sense of belonging, relationship with institutions, network density, strength of the network links, shared values, resource sharing, and assessment of innovations. The study population was composed of 108 third sector entities with the criterion of our study. The response rate was $48.35 \%$.

As part of the mixed-method design suggested by (Ugalde and Balbastre, 2013), personal interviews with seven stakeholders were conducted. The selection of informants was based on multi-reference suitability and included mainly representatives of and experts on the local third sector of social action. In parallel we conducted two focus groups, the first with staff from six local entities and the second with volunteers of five local entities. The data was analysed using SPSS v.19. and UCINETv.6 to generate visualizations of networks, and discourse analysis techniques (Fischer, 2003; Almaguer et al, 2014) were used to analyse narratives in the interviews and discussion groups.

\section{Findings}

In the case of Teruel, although there is a generalized sense of belonging to the sector and territory with $88.6 \%$ agreeing that their organization identifies with Teruel's third sector and $70.4 \%$ that entities in the province share values and principles, however it holds some distinctions in relation to the social system. For instance, the creation of independent local associations is positively regarded and proudly distinguished from the subsidiaries of larger organizations. Geographical identity creates a strong sense of attachment to the specific towns in which the organizations are located, to the Teruel province, and ultimately to the autonomous region. This strong identification with the local space creates an important characteristic in their collaboration with institutions and organizations.

In addition to the intensification of competition to provide goods and services, which affects the effectiveness of intervention of the third sector of social action, the results also suggest the fragmentation of smaller organizations in contrast to the increasing specialization of larger ones. In the focus group, it was explained that this differentiation is produced by the professionalization of larger organizations (having professional employers and volunteers with large trajectory) compared to small organizations that are not consolidated in their human resources capital. In their interviews, institutional actors suggested that the concept of third sector entities is more rooted in large than in small organizations due to the former holding a global perspective of the sector. This may also be the result of their professionalization through their employees and technicians, unlike small organizations that mainly use volunteers for their work and therefore invest less time and fewer resources in creating links and engaging in wider coalitions or networks through which strategic information is distributed.

Findings in the relational dimension suggest that characteristics and attributes of principal relationships and their incentives are constructed collectively rather than defined by specific organizations, with $72.7 \%$ of respondents believing that both a good and a bad reputation affects entities' relationships in the same sector, the same percentage $(72.7 \%)$ indicates that the main reason for establishing 
relationships with other entities is the pursuit of the same interests, rather than geographical proximity $(11.4 \%)$ or trust (11.4\%) as first hypothesized.

The survey results reveal that political and religious values are not necessarily shared with other organizations as a common denominator. However, in the network analysis we find complementary evidence of the network relationships. After performing various tests (estimation of the degree of centrality) on the network of 65 organizations (see figure 3), it was found that a set of entities plays an important role through its high level of collaboration with other entities, and that a particular multisectoral organization of religious origin conducts the most brokerage (i.e. the node that concentrates greatest density and flow of collaboration).

The mixed-method approach allowed us to explore other elements of the system. The interviews and discussion groups enabled in-depth investigation of the third-sector network's perceptions of its interactions and led us to the conclusion that the best-connected actors in such networks have a competitive advantage over those that are poorly-connected, actor's connection depends not only on their technological skills but also on other relational and symbolic capital. In this sense the networks established between organizations have strong internal cohesion but weak external connection. Our qualitative findings give more elements to explain the reasons for this trend: the latent meaning that actors give to their membership of and action in these networks has to do with promoting the sociability of its members, especially through volunteering. We conclude that there is a collective construction of social capital, that in this case, priories strengthening intrinsic networks within intra-territorial networks, over extrinsic, extraterritorial networks (i.e. across autonomous regions, the country or the continent). Face-to-face interaction, interpersonal trust and relationships play an important role in this construction of intrinsic networks. However new strategies and capitals are needed to reinforce the connection with extraterritorial networks.

Network analysis was relevant to understanding how the whole resources flows within the embedded network of social relations (Nahapiet and Ghoshal, 1998), and discourse analysis of the qualitative data allowed us to refine the answers and deepen the substantive features of the relations established between organizations as well as the importance of trust and reciprocity.

We have found that the network of third sector entities in the province of Teruel has a centralized structure, with three medium-sized entities acting as reference nodes for the cohesion of the network. Also the structure of the network of relationships between the third and the public sector is considerably denser (based on collaborative agreements and complementary action through concerted funding and grants, etc.) than that in the industrial sector. This correlates with the third sector's financial dependence on government institutions and its relevance as a central point of information to the majority of the actors.

KEY WORDS: Third social action sector, social capital, networks, trust, reciprocity. 


\section{1.- Introducción'1}

El diagnóstico del Tercer Sector de Acción Social (en adelante TSAS) (PwC, 2013) ha identificado diversos problemas del sector agudizados por el impacto de la actual reestructuración del Estado de Bienestar. Entre otros problemas se menciona la falta de propuestas para la gestión de las organizaciones (en un periodo de crisis) coherentes con su misión, sus principios y valores; la débil articulación entre lo territorial y lo estatal, que repercute tanto en la incidencia social como en la política; la inexistencia de criterios comunes o compartidos para la relación entre los diferentes agentes (organizaciones, empresas, medios de comunicación, sindicatos o universidades); la débil relación articulada con la sociedad civil y la intensificación de la competencia en la provisión de bienes y servicios que perjudican la eficacia de la intervención del TSAS.

La definición del Tercer Sector de Acción Social presenta dificultades por la existencia de unos límites difusos que propician diferentes interpretaciones (Rodríguez Cabrero y Marbán, 2008). En este trabajo hemos partido del concepto que se recoge en el II Plan Estratégico del Tercer Sector de Acción Social (2013-2016), que lo define como el ámbito formado por entidades privadas de carácter voluntario y sin ánimo de lucro que, surgidas de la libre iniciativa ciudadana, funcionan de forma autónoma y solidaria tratando, por medio de acciones de interés general, de impulsar el reconocimiento y el ejercicio de los derechos sociales, de lograr la cohesión y la inclusión social en todas sus dimensiones y de evitar que determinados colectivos sociales queden excluidos de unos niveles suficientes de bienestar.

Ariño Villarroya (2008:114) sostiene que el Tercer Sector español se caracteriza por su "raquítica coordinación interasociativa" producto de una alta heterogeneidad, disparidad estructural, dependencia financiera y baja articulación. Dicha estructuración la atribuye a la existencia de dos perfiles de asociaciones, con identidades diferenciadas. Por una parte, una identidad explícita heterocentrada (que hace suya los valores y principios de la solidaridad y el altruismo), y por otra, una identidad implícita autocentrada (que se vincula al grupo, a las relaciones sociales y al territorio en el que desarrolla su actividad). Sugiere que, de acuerdo con dicha tipología, en el caso español prevalece la segunda, ya que el deseo de "mantener relaciones sociales gratificantes y practicar una determinada actividad social en compañía de otras personas" está más generalizadas que las orientadas a la solidaridad y el altruismo. Sin embargo, es evidente que más investigación empírica es necesaria para entender la estructuración del sector y su manifestación en contextos sociales concretos. De particular importan-

1.- Este Trabajo se ha desarrollado en el marco del proyecto 2014/B015, financiado por la Fundación Universitaria Antonio Gargallo, y del Grupo de investigación consolidado S-64, financiado por el Gobierno de Aragón. Los autores agradecen las sugerencias y comentarios realizados por los evualuadores anónimos, que han contribuido a la mejora del trabajo. Todos los errores son responsabilidad de los autores. 
cia es en espacios rurales, donde este sector juega un papel importante en el reconocimiento y el ejercicio de los derechos sociales, y donde puede fortalecer la cohesión y la inclusión social.

El diagnóstico de Ariño Villarroya (2008) es compartido, en parte, por el trabajo de Aldashev, Marini y Verdier (2015) quienes, a nivel internacional, concluyen que el sector se caracteriza por una desconexión entre las entidades que financian la actividad de estas organizaciones y sus beneficiarios y usuarios finales. En ese marco, el II Plan Estratégico del Tercer Sector de Acción Social (Plataforma de ONG de Acción Social, 2013) plantea dos objetivos para abordar esos retos. Por una parte, garantizar la mejora de las condiciones en las que se da la prestación de bienes y servicios a personas y colectivos más vulnerables. Y por otra, aumentar la incidencia política y social del Sector a partir de su consolidación como actor social.

Ambos objetivos, sin embargo, deben considerarse en la particularidad del sistema social (Beer, 1977). Esa totalidad relativa que es el conjunto de relaciones existentes y sus ámbitos de interacción e influencia, configura un sistema dinámico y complejo, al estar compuesto de procesos adaptativos y cambiantes que mantienen su existencia ante estados de perturbación o inestabilidad mediante procesos de autorregulación y retroalimentación (García, 2006). Consideramos que entender el sistema social en el que se inserta permitirá entender las dificultades y oportunidades que las TSAS tienen en su contexto específico (organizacional, cultural y geográfico) para responder a esos retos. Las organizaciones del TSAS se caracterizan por su capacidad de generar un resultado diferente en la dimensión macro, meso y micro de la vida social (Bassi y Vicenti, 2015).

En este trabajo nos hemos centrado en el estudio del TSAS de la provincia de Teruel (España). En la categoría de Flybjerg (2004) consideramos el caso de Teruel como un caso extremo porque sus rasgos particulares de distribución geográfica y desertificación poblacional hacen especialmente relevante al sector del TSAS. A esas particularidades se le suma una espiral decadente donde se vuelve particularmente difícil de mantener el gasto público en servicios sociales particularmente en zonas geográficamente dispersas y poblacionalmente reducidas. Ante ese contexto y por su configuración particular, las organizaciones del TSAS tienen retos importantes para llevar a cabo sus objetivos de intervención social.

Tal y como mencionan Peraldi \& Rombaldi (2009) analizando el caso de Córcega, los territorios rurales fuertemente debilitados sufren problemas importantes de desarrollo. En este contexto, es fundamental una mejor integración de las organizaciones sociales en estos territorios, pero para que tal evolución tenga lugar, es conveniente que la utilidad social producida por estas organizaciones se reconozca socialmente. Nosotros argumentaremos que además, es necesario que el sector reconozca su propia estructura de red de relaciones identificando sus fortalezas y debilidades.

Por ello, este trabajo busca analizar la perspectiva de miembros representativos, diagnosticar los problemas comunes y proponer acciones derivadas de sus propias reflexiones respondiendo a las siguientes preguntas: ¿cuál es la caracterización del TSAS en el caso de Teruel, con sus particulari- 
dades geográficas y poblacionales?, ¿cómo se configuran las diferentes dimensiones del capital social y cómo se expresa a través de sus redes sociales? y ¿cuáles son los problemas del TSAS en un contexto como el de Teruel? Buscamos que nuestra contribución permita caracterizar el tipo de capital social existente en el TSAS turolense para que los responsables de las entidades y del bienestar social pueden identificarse en mejores condiciones para entender y fortalecer su capital social.

\section{2.- Capital social en redes sociales}

El concepto de capital social ha adquirido una gran relevancia y es reconocido como un elemento importante para las organizaciones debido a su contribución a la ventaja competitiva, recibiendo una creciente atención en la literatura de dirección estratégica desde hace más de dos décadas (Nahapiet y Ghoshal, 1998; Tsai y Ghoshal, 1998; Adler y Kwon, 2002; McFadyen y Cannella, 2004; Hitt, Ireland, Sirmon \& Trahms, 2011; Hollenbeck, \& Jamieson, 2015). Se considera que es un recurso arraigado en la estructura social de los individuos y que se genera a través de la interacción (Lin, 1999; Adler y Kwon, 2002; De la Garza Martínez, López y Rojas, 2012); y que posee unas características propias en contextos rurales (Peraldi y Rombaldi, 2009; Buciega y Esparcia, 2013; Debertin y Goetz, 2015; David y Tacoronte, 2015). Aunque no hay consenso en una definición precisa, podemos definir el capital social de un actor como el conjunto de recursos arraigados dentro de su red de relaciones sociales, además de todos los recursos accesibles a través de esa misma red (Nahapiet y Ghoshal, 1998).

Sabemos que el capital social es un constructo multidimensional, por lo que para su análisis es necesaria la identificación y medida de una serie de dimensiones (Koka y Prescott, 2002); no es "propiedad individual" ni puede monetizarse su valor al igual que otras formas de capital (Bourdieu, 1985). En lugar de ello, facilita las relaciones entre los agentes y los une a través de redes de confianza (Anderson y Jack, 2002; Paldam, 2000). Los estudios en este campo sugieren que el capital social resuelve problemas de coordinación, reduce los costes de transacción, facilita el flujo de información entre y a través de diversos individuos (Lin, 2001); y, además, contribuye de forma significativa a las ventajas de la organización, ya que permite desarrollar el aprendizaje y transmitir el conocimiento en la misma (Tsai y Ghoshal, 1998; Adler y Kwon, 2002; Carey, Lawson y Krause, 2011).

Natal y Chávez (2010) analizan los trabajos académicos que relacionan capital social y Tercer Sector. Plantean una perspectiva crítica según la cual el Tercer Sector complementa o sustituye al Estado en cuestiones sociales y advierten que ese enfoque propone que la acción civil voluntaria, tanto económica como política, sea la ruta para atender una problemática social cada vez más compleja. Esa visión, continúan Natal y Chávez (2010), cree que un Estado reducido debe fomentar la participación del Tercer Sector en el mercado de servicios, en la lucha contra el desempleo y en contribuir a resolver algunas deficiencias sociales como la pobreza. 
Los teóricos del capital social están de acuerdo en que los actores mejor conectados en su red poseen una ventaja competitiva sobre los pobremente conectados. La perspectiva de redes nace como contraste a una visión atomística, que asume que las organizaciones actúan solas y se entiende como la perspectiva del arraigo (embeddedness). Marcuello et al. (2010) sugieren que para una entidad social -sea fundación o asociación- el trabajo en red es una necesidad y una seña de identidad. Así, la creación de plataformas, federaciones y/o coordinadoras cumple con ese objetivo de distinción y referencia. Son espacios de cooperación, de encuentro, de debate y fortalecimiento de las entidades que junto a otros, con sus "pares", se posicionan en la sociedad (Marcuello et al, 2010). Sin embargo no hay tanto consenso respecto a qué significa estar mejor conectados y cómo se define el uso de los vínculos desde un grupo específico, particularmente en contextos rurales donde el capital social juega un papel crucial.

Existen aproximaciones que nos sugieren que el capital social que posee un actor/organización, debe analizarse desde la perspectiva de la forma en que accede a los recursos (económicos, informacionales, simbólicos) a través de su red de relaciones sociales y en forma de capacidades organizativas arraigadas en esa red. Cuando se habla de una red, se entiende como un grupo de individuos que, en forma agrupada o individual, se relacionan con otros con un fin específico. Las redes pueden tener muchos o pocos actores y una o más clases de relaciones entre pares de actores organizados en los siguientes tipos: (i) Nodos 0 actores, personas o grupos de personas que se encuentran en torno a un objetivo común cuya suma de todos los nodos representa el tamaño de la red; (ii) Vínculos, relaciones que existen entre dos o más nodos y (iii) Flujo, que es la dirección del vínculo.

Casanueva y Galán (2002) catalogan la red y sus características principales, recurriendo a los siguientes indicadores: (a) Densidad, como el cociente resultante entre el número de nexos de la red y el número de relaciones totales posibles que se pueden establecer entre los agentes; (b) Centralidad, que mide el número de vínculos que tiene un nodo con el resto de miembros de la red sin diferenciar entre las relaciones establecidas por dos actores distintos (Hanneman, 2001) y (c) Intermediación, que identifica la medida que esté situado entre los caminos geodésicos de otros pares de nodos en la red (Hanneman, 2001). Este rol otorga centralidad a un actor, al que necesariamente deben recurrir otros si quieren establecer una conexión entre ellos, lo que les hace relativamente dependientes del primero (evitable mediante otras alternativas o rutas establecidas en la red, lo que resta importancia a este papel).

En este trabajo, siguiendo a Nahapiet y Ghoshal (1998) y a Carey, Lawson y Krause (2011) distinguimos entre las dimensiones estructural, relacional y cognitiva del capital social. La dimensión estructural se centra en las características de la red de relaciones establecidas, enfocándose particularmente en las interacciones sociales que se producen. Por su parte, la dimensión relacional hace referencia a las características y atributos de dichas relaciones y a los incentivos que se derivan de la relación continuada (Gulati et al., 2000); según Tsai y Ghoshal, (1998) aspectos clave de esta dimensión son la confianza relacional y las normas compartidas. Finalmente, la dimensión cognitiva representa los recursos proporcionados por el entendimiento y significado compartido entre los miembros 
de la red (Nahapiet y Ghoshal, 1998; Carey, Lawson y Krause, 2011; Chang y Chuang, 2011). Aspectos principales de esta dimensión son por ejemplo, la cultura y las metas compartidas entre los miembros de la red. Las metas compartidas representan el grado en el que los miembros de la red comparten un entendimiento. La cultura se transmite y comunica entre los miembros del grupo y sirve para interpretar y entender sus experiencias en común (Morgan, 1986).

Además de las dimensiones estructural, relacional y cognitiva del capital social, Camagni (2003) sugiere que la proximidad facilita las relaciones frecuentes y estrechas entre las organizaciones y en la medida que las organizaciones se encuentran vinculadas geográfica e históricamente, aumentará la continuidad de las interacciones. En este sentido, señala que algunos principios pueden influir y determinar las características y estructura de red en la que las entidades se encuentran arraigadas. Esos principios permitirían establecer una red cohesiva, densa y con fuertes vínculos por tener, por ejemplo, un territorio geográfico compartido.

Para esta investigación, tomamos como punto de partida el trabajo clásico sobre el arraigo de Granovetter (1985). Su argumento del arraigo sugiere que la conducta y las organizaciones están afectadas por relaciones continuas y que éstas no pueden entenderse de forma separada o independiente. La aproximación de Granovetter acentúa el papel que pueden desempeñar las relaciones sociales en la generación de la confianza y en limitar o desalentar la desconfianza entre las partes (Oinas, 1998). Por su parte, Uzzi (1997), sugiere que las relaciones arraigadas presentan tres componentes principales que regulan las expectativas y los comportamientos de los intercambios entre los socios: a) la confianza, b) la transferencia de información estratégica y c) los acuerdos conjuntos para la resolución de problemas. Dado que las relaciones sociales que constituyen el capital social, ocurren y pueden ser estudiadas a diversos niveles, desde el individual hasta el de la sociedad en su conjunto.

\section{3.- Teruel: un estudio de caso extremo}

Elegimos el estudio de caso como la base del diseño metodológico, en primer lugar, porque una de sus principales ventajas es su idoneidad para alcanzar un profundo nivel de comprensión de los procesos sociales contemporáneos (Yin, 1993, 2003) y segundo, porque puede incorporar una variedad de técnicas de investigación y fuentes de prueba. Esto es particularmente atractivo para quieres desean realizar un "análisis de múltiples niveles o componentes" (Vaus, 2001: 220).

Como estudio de caso elegimos el TSAS de Teruel, provincia de Aragón, España. Aragón representa el 2,9 de la población total de España y el 9,4\% del territorio nacional. La combinación de ambos factores, población y territorio, configura una superficie poco poblada con una tasa de densidad de pobla- 
ción de 28,2 habitantes por $\mathrm{Km}^{2}$, mientras que en el conjunto de España la densidad es de 93,3 hab/ $/ \mathrm{Km}^{2}$ y en la UE-27 de 116 hab/km². Según datos del Instituto Aragonés de Estadística (IAEST), la población de Aragón se cifra en 1.347.150 habitantes, mientras que la de Teruel es de 142.183 personas, de las cuales 72.529 son hombres y 69.654 son mujeres ${ }^{2}$. Otros aspectos hacen particular esta provincia seleccionada. Así, en Teruel, el $95 \%$ del territorio y el $46,7 \%$ de la población tiene características rurales (Rubio, 1996), estando dividida en 236 municipios (Ver figura 1 y 2 para referencia geográfica).

\section{Figura 1 y 2. Localización de Aragón en el contexto español (Hansen y Mutxamel, 2009) y división geográfica de la provincia de Teruel (Miguillen 2010)}
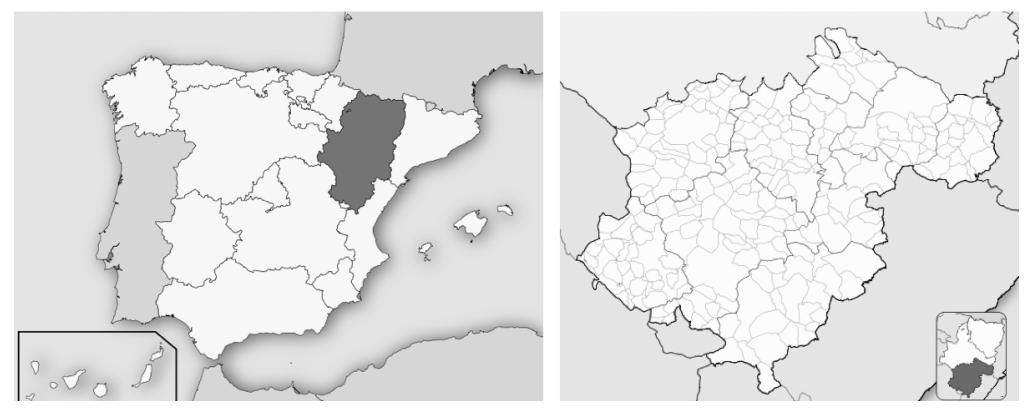

El tema de la sostenibilidad de los servicios sociales en estos espacios donde se combina despoblamiento y envejecimiento es delicado y urgente. La edad media más alta de la población aragonesa corresponde a la provincia de Teruel, con 45,1 años ${ }^{3}$. Del total de municipios, el $78 \%$ tienen un grado de envejecimiento superior al 25\% (Mur, 1998).

El mapa comarcal de Teruel también presenta unos índices muy bajos de población. Solo dos núcleos superan los cinco mil habitantes: Alcañiz, con 15.669 y Andorra, con 8.147. 50.801 personas viven en poblaciones de menos de 1000 habitantes. Seis de las diez comarcas que forman la provincia de Teruel no alcanzan los 10.000 habitantes (Cuencas Mineras, 8.833; Andorra-Sierra de Arcos, 11.113; Bajo Aragón 29.875; Comunidad de Teruel, 47.185; Maestrazgo, 3.464; Sierra de Albarracín, 4.783; Gúdar-Javalambre, 8.095; Matarraña, 8.630; Jiloca, 13.435; Bajo Martín, 6.768), siendo las menos pobladas las que han sufrido una mayor disminución de población en el último quinquenio. La densidad demográfica de Teruel contrasta datos históricos de 1960 (16 habitantes $/ \mathrm{km} 2$ ) a los de 2000 (9 habitantes $/ \mathrm{km} 2$ ). Esto quiere decir que la población actual representa solo un $57 \%$ de la existente en 1960. Teruel es la provincia española que ha experimentado una mayor pérdida demográfica (Collantes y Pinilla, 2003).

2.- Cifras oficiales de población. Padrón a 1 de enero de 2013. Real Decreto 1016/2013, de 20 de diciembre, por el que se declaran oficiales las cifras de población resultantes de la revisión del Padrón municipal referidas al 1 de enero de 2013.

3.- Fuente: Informe socio-económico CESA 2012. 
Al elevado número de municipios de la provincia de Teruel (236, como ya se ha mencionado), junto a su escasa población y su elevada tasa de sobreenvejecimiento, se añade la pérdida de capacidad de renovación, al no haber población para reemplazar la existente. Ante ese panorama al que se le suma la reducción de gasto público en servicios sociales, las organizaciones del TSAS tienen retos importantes para llevar a cabo sus objetivos de intervención social, por lo que se antoja necesaria una estrategia que les permita ser eficaces en su organización interna y con sus pares.

Flyvbjerg (2004) defiende la proximidad del estudio de casos con las situaciones de la vida real y su gran riqueza de aspectos importantes para el desarrollo de una visión matizada de la realidad (Flyvbjerg, 2004:38). Este método permite además obtener experiencias concretas por medio de la proximidad continuada con la realidad estudiada y de la retroalimentación de quienes están siendo estudiados. En la categoría de Flybjerg consideramos el caso de Teruel como un caso extremo (Flyvbjerg, 2004:45) porque sus rasgos particulares de distribución geográfica y desertificación poblacional hace especialmente estratégico y relevante al sector del TSAS.

Como estudio de caso único seguimos las recomendaciones de Lipset y Trow (2004), considerando un diseño metodológico mixto que permitiera reforzar los aspectos de validez y para establecer criterios de rigor cualitativo (Baxter y Eyles, 1997). Para ello nos basamos en un enfoque metodológico mixto que integra instrumentos cuantitativos y cualitativos de recolección de datos. Tal y como señalan Ugalde y Balbastre (2013), las ventajas de este enfoque es que permite obtener inferencias más fuertes y la compensación de las desventajas que existen en las metodologías cualitativas y cuantitativas cuando se utilizan individualmente (Molina, 2010). Los beneficios de estos tipos de diseño son hallazgos complementarios que ofrecen una aproximación desde diferentes ángulos al fenómeno analizado. Consideramos que integrando esta perspectiva al estudio de caso se puede ofrecer una aproximación metodológica que aproveche las fortalezas de las técnicas de investigación seleccionadas, en este caso combinando un diseño de encuestas a las entidades del TSAS de Teruel y complementando con entrevistas a actores clave y dos grupos de discusión tanto a técnicos como voluntarios del sector. Del mismo modo hemos considerado las observaciones pertinentes de Yaojun Li (2015) sobre los métodos y estrategias para el estudio del capital social.

\section{4.- Aspectos metodológicos}

En el contexto de esta investigación, nos centramos en las organizaciones objeto de estudio que se ubican en la provincia de Teruel (figura 1), entidades que pertenecen al Tercer Sector de Acción Social formadas cada una por un conjunto de asociados o socios y sus relaciones institucionales, para la persecución de un fin de forma estable, sin ánimo de lucro y con una gestión democrática. 
Considerando la especificidad de las organizaciones más pequeñas del TSAS, poco visibles en el espacio social y con necesidades y objetivos diferenciados, se consideró la posibilidad de realizar una encuesta a estas organizaciones, con el objetivo de que hubiera una representación específica y significativa de la opinión e intereses de estas organizaciones en el análisis situacional para el plan estratégico.

Para los fines de este estudio, se diseñó una encuesta integrada por 58 preguntas distribuidas en 9 apartados y permitieron recabar información sobre variables relacionadas con el capital social existente en el sector: sentimiento de pertenencia, relación con instituciones, densidad de la red, fortaleza de los vínculos, normas y valores compartidos, riqueza del intercambio, beneficios y recursos nuevos exclusivos y valoración de las innovaciones.

Para establecer el universo de estudio, se integraron los datos disponibles en los registros oficiales de la Diputación General de Aragón (DGA), Ayuntamientos y Comarcas de la provincia de Teruel para elaborar un directorio actualizado de las entidades del TSAS que representaran la totalidad del universo a estudiar. Se obtuvo inicialmente un listado de 232 entidades, que fue posteriormente corroborado mediante contacto con los datos proporcionados, identificando además el enlace idóneo en cada entidad para la realización del estudio. Debido a que algunos datos se repetían y otros correspondían a organizaciones que ya no existían, la población objeto de estudio quedó constituida por 108 entidades teniendo como criterio central aquellas que tuvieran un ámbito territorial local.

Se hizo una prueba piloto del cuestionario con cuatro entidades seleccionadas y a la coordinadora aragonesa de voluntariado. Una vez mostrados los fallos de la encuesta, se corrigieron y se realizó el cuestionario definitivo. La encuesta fue distribuida mediante correo electrónico para que pudiera ser respondida a través de internet y en el caso de la no respuesta se contactó telefónicamente. El cuestionario fue enviado varias veces en el periodo del 24 de marzo al 17 de abril de 2014. Se hizo un seguimiento telefónico en dos cohortes para incidir en su respuesta. La tasa de respuesta ha sido de un $48,35 \%$.

Como parte de un diseño metodológico mixto (Ugalde y Balbastre, 2013) se realizaron entrevistas personales a nueve informantes clave, de entidades e instituciones. La selección de los informantes se hizo basada en la idoneidad multireferenciada, es decir, se les preguntó a las propias entidades que identificaran a personas con una reconocida trayectoria en el sector, de tal manera que los nombres sugeridos más reiterados determinaron la muestra, que fueron principalmente representantes 0 expertas del TSAS turolense, con los perfiles que cubrieran un espectro más amplio ${ }^{4}$. Los temas

4.- Experto en voluntariado social y redes de trabajo del tercer sector de acción social, representante de entidad referente al voluntariado y además socia de una asociación referente al tercer sector de acción social, persona experta en Servicios Sociales, gerente de entidad a nivel local y experta en intervención social, experta en participación social Técnico, representante de una entidad social de la provincia de Teruel, responsable de Servicios Sociales Comarca de Gudar-Javalambre, Gerente de una pequeña entidad y persona experta en intervención social, Directora del área de Servicios Sociales Comarca de Teruel. 
fueron abordados en relación a los aspectos que se cuestionaban en la encuesta con el fin de complementar la información empírica con una perspectiva cualitativa. Paralelamente se realizaron dos grupos de discusión, el primero formado por representantes de seis entidades con técnicos de las organizaciones, y el segundo formado por otras cinco entidades, con un perfil de voluntarios dentro de las organizaciones, todas provenientes de la provincia de Teruel. La duración aproximada de los dos grupos de discusión fue de unos 60 minutos. En la tipificación de los grupos de discusión tuvimos en cuenta el trabajo de Ariza, Fernández y Tirado (2016) sobre los principales perfiles del voluntariado.

Tanto las entrevistas como los grupos de discusión nos permitieron profundizar en las cuestiones que se abordaban en el cuestionario on-line. Las entrevistas fueron codificadas en orden cronológico de realización de la técnica $(E 1, E 2, \ldots)$. En la fase de análisis, se procedió a la explotación de Ios datos recabados, para lo que se analizaron los cuestionarios a través de SPSS v.19. Los datos cualitativos fueron analizados mediante la técnica de análisis del discurso (Fischer, 2003; Almaguer et al, 2014). También se aplicó el análisis de redes al conjunto de relaciones entre agentes. Esta metodología ha sido propuesta por distintos autores como adecuada para el estudio de las redes. Algunos como Cuñat (2015) ha analizado las redes en el surgimiento y consolidación de las cooperativas de trabajo asociado. Esta metodología se basa en el supuesto de que el comportamiento de los individuos no solo depende de sus atributos o características, sino también de su participación en relaciones sociales estructuradas. Para la generación de visualizaciones de las redes se utilizó el programa UCINETv.6. Los resultados de ese análisis mixto se presentan en las siguientes secciones.

\section{5.- Dimensión estructural del TSAS de Teruel}

De la población de 108 entidades, se obtuvo una tasa de respuesta de 48,35\%. La pregunta relativa al ámbito de actuación era de respuesta múltiple y a la entidad se le permite seleccionar diferentes ámbitos de actuación. De acuerdo a estas respuestas ${ }^{5}$, el ámbito de actuación de las entidades fue el siguiente: acción social $(75 \%)$, discapacidad $(52,3 \%)$, salud (52,3\%), juventud (45,5\%), exclusión social $(45,5 \%)$, mujer $(43,2 \%)$, ocio y tiempo libre $(43,2 \%)$, infancia $(31,8 \%)$, personas mayores $(31,8 \%)$, justicia y derechos humanos $(29,5 \%)$, cooperación al desarrollo (25\%), inmigración $(20,5 \%)$, vivienda $(18,2 \%)$, medioambiente $(18,2 \%)$.

El perfil de los miembros y voluntarios que pertenecen a estas organizaciones es heterogéneo. En cuanto a la variable "edad" encontramos diferencias significativas. Mientras que los más jóvenes atien-

5.- La pregunta relativa al ámbito de actuación es de respuesta múltiple y a la entidad se le permite seleccionar diferentes ámbitos de actuación. 
den situaciones relacionadas con la inmigración, la discapacidad, la infancia y los derechos humanos, los adultos y mayores se ocupan de la atención a la tercera edad, el ocio y tiempo libre y exclusión social. En el gráfico 1 se muestra el intervalo de las edades de los miembros y voluntarios en cada ámbito de actuación considerado.

\section{Gráfico 1. Ámbitos de actuación y edad}

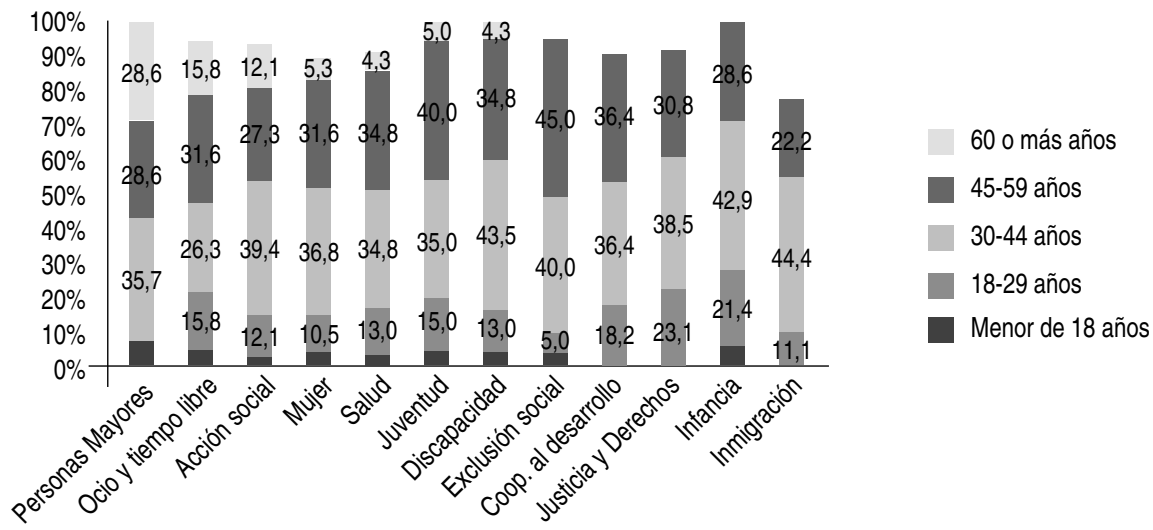

FUENTE: Elaboración Propia.

Cabe destacar que las personas menores de 18 años casi no forman parte del ámbito de actuación del TSAS, predominando las personas entre 30-44 años y entre 45 y 59 años. Consideramos que la edad promedio de las organizaciones es importante de cara a los retos organizacionales, tecnológicos e informacionales a los que se enfrentan las entidades del tercer sector, tal y como veremos en los siguientes apartados.

\section{6.- Arraigo al sector $y$ al territorio}

Con respecto al sentimiento de pertenencia e identidad con el TSAS, del total de las encuestas contestadas, el 88,6\% están de acuerdo con que su organización se identifica con el TSAS de la provincia de Teruel y el 70,4\% también apuntan a que sí se comparten principios entre entidades que se encuentran en la provincia de Teruel. 


\section{Tabla 1. Sentimiento de pertenencia al tercer sector de acción social}

\begin{tabular}{|l|ccccc|}
\hline & $\begin{array}{c}\text { Totalmente } \\
\text { en desacuerdo }\end{array}$ & $\begin{array}{c}\text { En } \\
\text { desacuerdo }\end{array}$ & $\begin{array}{c}\text { Ni de acuerdo } \\
\text { ni en desacuerdo }\end{array}$ & $\begin{array}{c}\text { De } \\
\text { acuerdo }\end{array}$ & $\begin{array}{c}\text { Totalmente } \\
\text { de acuerdo }\end{array}$ \\
\hline $\begin{array}{l}\text { Considero que mi organización } \\
\text { se identifica con el TSAS de la } \\
\text { provincia de Teruel }\end{array}$ & $0,0 \%$ & $4,5 \%$ & $6,8 \%$ & $38,6 \%$ & $50,0 \%$ \\
\hline $\begin{array}{l}\text { Cree que se comparten ciertos principios } \\
\text { entre las entidades que se encuentran } \\
\text { en la provincia de Teruel }\end{array}$ & $4,5 \%$ & $9,1 \%$ & $15,9 \%$ & $54,5 \%$ & $15,9 \%$ \\
\hline
\end{tabular}

FUENTE: Elaboración Propia.

Así, si bien, es generalizada una sensación de pertenencia al sector y al territorio, la posición dentro de ese sistema social tiene distinciones particulares. La creación de organizaciones locales independientes de las centrales es bien vista por el resto de las organizaciones territoriales y motivo de orgullo de la propia organización. Esto sugiere una expresión del alto sentido de pertenencia organizacional y territorial, que se manifestó enlas entrevistas y grupos de discusión como un elemento clave en la vinculación de las relaciones sociales forjadas en y para el propio territorio. Entidades que trabajan a nivel provincial pero pertenecen a una estructura nacional son diferenciadas por tener "objetivos a nivel provincial pero que responden a unos objetivos nacionales". Este tipo de organizaciones las distinguen de aquellas que surgen a partir de necesidades concretas locales. En algunos casos se percibe una centralización en la capital de la Comunidad Autónoma (Zaragoza). Algunos refieren el origen de su organización como una declaración de autonomía, en tanto que la organización central era la que prescribía las actividades y centralizaba los recursos.

"...tuvimos que hacer nuestra propia asociación porque no nos dejaban trabajar.... Es decir, todo estaba centralizado en Zaragoza: el congreso, las subvenciones apoyaban los gastos de Zaragoza, las visitas priorizaban allá..." (E1)

En las entrevistas, los actores institucionales sugerían que el concepto de entidad del Tercer Sector está más arraigado en entidades grandes que en las pequeñas, en tanto que en las primeras estaba interiorizada una visión más global del sector. Un factor al que le atribuían esta distinción era la profesionalización de la entidad a través de la contratación de empleados y técnicos a diferencia de las pequeñas que se sostienen principalmente del trabajo voluntario de sus socios, quienes tienen problemáticas concretas que tratan de solventar en su tiempo de acción y por esas mismas limitaciones dedican menos tiempo y recursos a la creación de vínculos estratégicos.

Un primer punto de análisis fue el sentido de pertenencia, tanto geográfica como al sector de actuación. Con respecto a la identidad geográfica, prevalece una fuerte percepción de arraigo a su espa- 
cio local más cercano. Esa identificación es primordialmente hacia el territorio local, luego hacia la provincia, y en última instancia a la comunidad autónoma. Esa fuerte identificación con el espacio local genera una distinción a la hora de colaborar con instituciones y organizaciones. Aunque algunos actores colaboran entre municipios vecinos que no necesariamente son de la misma comarca o provincia demostrando la permeabilidad de sus fronteras territoriales, los límites geográficos siguen siendo altamente significativos a la hora de planear intensificar y/o aumentar sus zonas de influencia. El énfasis en la localidad sugiere un arraigo diferenciante de las entidades, tal y como se mencionó en un grupo de discusión:

"....los de la capital de la provincia (Teruel capital) hacia la ciudad... las entidades de Alcorisa son de Alcorisa y las de Teruel son las de Teruel ciudad. Matarraña tiene una identidad propia, la perspectiva la tienen de provincia pero se tienen más identificados con lo local o como mucho a nivel comarcal..." (E7)

Ese arraigo puede considerarse como una fuerte identidad hacia el espacio local, pero también como una atomización en el mismo. Eso se infiere en la distinción que se hace de aquellas entidades que logran una colaboración más allá de su municipio o incluso de la provincia. Por ejemplo, el caso de Andorra o Alcañiz, de quienes refirieron en el grupo de discusión de técnicos del TSAS

“...No tiene una mentalidad provincial, son un foco de influencia." (GD1)

Sin embargo, se señaló que los límites geográficos a nivel institucional están más delimitados que a nivel de tercer sector. Es decir, el tercer sector puede ser más permeable a las fronteras territoriales pues no tiene limitaciones administrativas como las instituciones. Los propios actores institucionales entrevistados dijeron que las instituciones podrían aprovechar más las relaciones existentes entre las organizaciones con el fin de poder llegar a otras zonas geográficas. Esta puede ser entonces un área de oportunidad tanto para el TSAS como para las instituciones con las que interactúan.

\section{7.- Valores compartidos}

Los valores que se comparten en una comunidad son parte del capital social que les permite funcionar. Al preguntar si se consideraba que compartir valores favorecía la interacción entre entidades e instituciones, el $93,2 \%$ de los encuestados contestó afirmativamente a esa pregunta, frente a un $6,8 \%$ que indicó que compartir valores no favorecía la interacción entre entidades e instituciones. De los 49 casos encuestados, el $95,5 \%$ opina que los principios que más se comparten son la solidaridad, la participación, la responsabilidad y el compromiso. Contrasta que tanto valores políticos como religiosos fueron los que tuvieron porcentajes más bajos en esta respuesta (ver tabla 2). 
Tabla 2. Principios que se comparten entre entidades

\begin{tabular}{|lcc|}
\hline Principios & $\mathrm{SI}$ & $\mathrm{NO}$ \\
\hline Políticos & $13,6 \%$ & $72,7 \%$ \\
Religiosos & $18,2 \%$ & $68,2 \%$ \\
Rendición de cuentas & $68,2 \%$ & $22,7 \%$ \\
Transparencia & $79,5 \%$ & $13,6 \%$ \\
Democracia & $86,4 \%$ & $11,4 \%$ \\
Igualdad & $86,4 \%$ & $11,4 \%$ \\
Compromiso & $88,6 \%$ & $9,1 \%$ \\
Responsabilidad & $88,6 \%$ & $6,8 \%$ \\
Participación & $88,6 \%$ & $9,1 \%$ \\
Solidaridad & $95,5 \%$ & $4,5 \%$ \\
\hline
\end{tabular}

FUENTE: Elaboración Propia.

En los resultados de la encuesta se obtiene que los valores políticos y religiosos no necesariamente se comparten con otras organizaciones como común denominador. Sin embargo, en el análisis de redes constatamos otras evidencias complementarias. La figura 3 presenta la red de relaciones entre entidades que se relacionan habitualmente. El total de componentes en esta red fue de 65 actores.

\section{Figura 3. Red de relaciones entre entidades que se relacionan habitualmente}

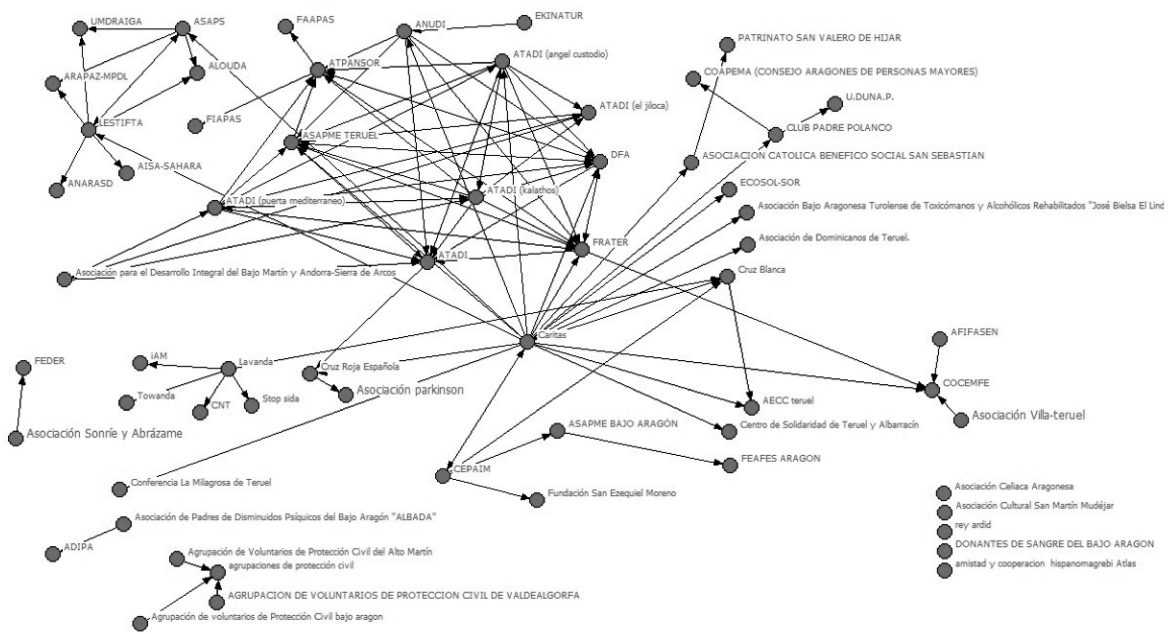

FUENTE: Elaboración Propia. 
Aunque a simple vista parece obvio que se trata de una red densa donde existen nodos con mayor relación que otros, tras realizar diversas pruebas (estimación del grado de centralidad), se constató que existe un conjunto de entidades que juegan un papel importante por su alto nivel de colaboración con el resto de las entidades. ${ }^{5}$ Si bien, la limitación de este tipo de esquemas es que los nombres de los nodos pierden especificidad, al menos da una idea de las relaciones entre los principales nodos. Los resultados de intermediación concluyen que una organización multisectorial de origen religioso (Cáritas), es el actor de mayor intermediación, es decir uno de los nodos que concentra mayor densidad y flujo de colaboración, en tanto que es la entidad que más pares de nodos conecta entre sí. Esto puede explicarse también por el flujo de recursos e información con el que puede contar una organización con mayor estructuración y vinculación fuera del territorio, comparado con otras más pequeñas y autónomas.

Destaca asimismo que existen cinco nodos que están enteramente desconectados de la red, así como tres grupos que colaboran entre ellos pero no tienen conexión con el resto del sistema. La densidad, que indica la proporción de relaciones que se dan respecto al total de posibilidades, es del $2,45 \%$. Se trata de una densidad baja, debido a que en este cálculo hemos considerado la densidad según la concentración de menciones o referencias por parte otras entidades. En ese sentido, las entidades que solo son nombradas por una entidad presentan una baja densidad de relaciones.

A través del análisis de redes se modeló también la red de relaciones entre entidades que se relacionan esporádicamente. Cada actor está representado con un nodo (un círculo rojo en este caso), mientras que la flecha que sale de cada actor dirigiéndose a otro indica la relación que tiene de manera esporádica con esa entidad (ver Figura 4).

\section{Figura 4. Red de relaciones entre entidades que se relacionan esporádicamente}

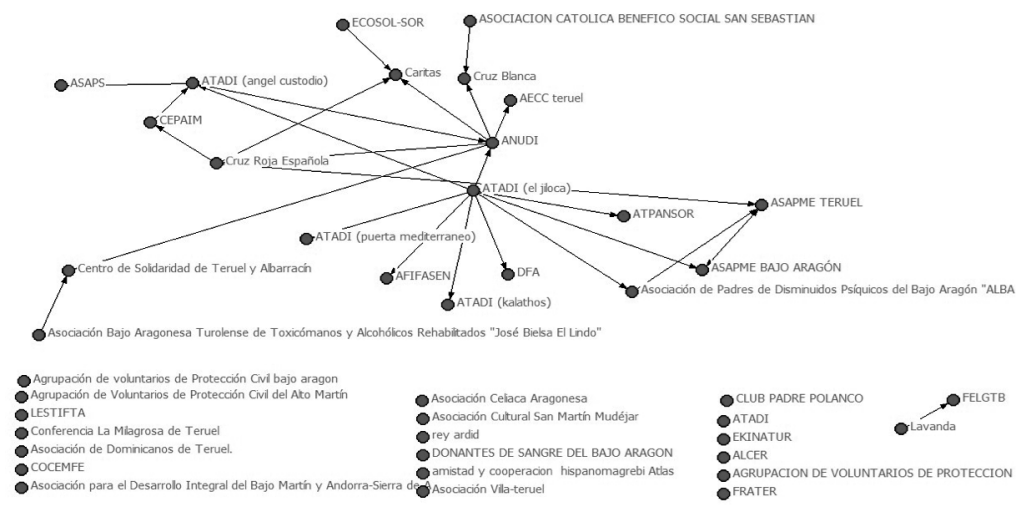

FUENTE: Elaboración Propia.

5.- Se trata de las siguientes entidades: ATADI, ATPANSOR, ASAPME Teruel, FRATER y DFA, cuyo ámbito de actuación es la discapacidad; se reúnen de forma periódica a través de lo que denominan grupo "Avanzamos". 
Esta red presenta los resultados obtenidos y su objeto de estudio está compuesto por 47 actores. Tras realizar diversas pruebas, como fue el grado de centralidad, se constató que las entidades como Cáritas y ASAPME Teruel vuelven a jugar un papel importante por su alto nivel de colaboración con el resto de las entidades. Los resultados de intermediación nos demuestran que ANUDI es el actor de mayor intermediación: es decir, en esta red, ANUDI es la entidad que más pares de nodos puede conectar entre sí.

En esta representación de relaciones esporádicas, destaca que la mitad de los nodos pertenecientes a esta red están enteramente desconectados de ella, así como dos grupos que colaboran entre ellos pero no tienen conexión con el resto del sistema. La densidad, que indica la proporción de relaciones que se dan respecto al total de posibilidades, es del 2,82\%. Hay también, por lo tanto, una baja densidad debido a que en este cálculo hemos considerado entidades que solo son nombradas por una entidad y que nadie más vuelve a mencionar y que muchos de los actores (nodos) están desconectados.

\section{8.- La dimensión relacional de las redes en el TSAS turolense}

La dimensión relacional hace referencia a las características y atributos de las relaciones y los incentivos que se derivan de la relación continuada (Gulati et al., 2000). Aspectos clave de esta dimensión son la confianza relacional y las normas compartidas (Tsai y Ghoshal, 1998). Cinco preguntas del cuestionario realizado fueron dedicadas a analizar las normas y valores compartidos en el TSAS.

La primera pregunta analizó el grado de confianza entre entidades, sin mostrar los resultados una tendencia concluyente. Por un lado, un $36,3 \%$ de los encuestados consideran que sí hay confianza entre las propias entidades del sector, mientras que un $15,9 \%$ opina lo contrario. El consenso se construyó alrededor de la dimensión de la valoración simbólica del sistema social sobre la actuación de la organización. Un $72,7 \%$ de los encuestados consideran que tanto la buena como la mala reputación afecta a las entidades dentro del mismo sector. En la tercera pregunta, referida a la reciprocidad y ayuda mutua entre las entidades, el $61,4 \%$ de las encuestas respondidas opinaron que sí existe esta ayuda mutua, mientras que un $31,8 \%$ no se pronunció sobre esta cuestión.

La última pregunta indaga por la existencia de normas o reglas no escritas que regulen el sector, refiriéndonos con el término "no escritas" a códigos de buenas prácticas que se dan por supuesto en organizaciones dedicadas a ayudar a los demás de forma altruista. En este sentido, el $40,9 \%$ de los encuestados no están ni de acuerdo ni en desacuerdo, y el 34,1\% están de acuerdo con esta afirmación. Los resultados de las encuestas parecen ser no significativas en relación a esta cuestión, así 
como a la pregunta sobre si se planteaba castigar o sancionar a las entidades que se comportasen de forma oportunista; el 45,5\% afirmó que no estaba de acuerdo ni en desacuerdo. Tampoco en los grupos de discusión o entrevistas se encontraron resultados significativos, probablemente porque hasta la fecha, las organizaciones nunca se hayan encontrado con una situación de esta índole. En una entrevista a actores institucionales se menciona:

"...Yo eso no lo he visto nunca, me decantaría porque no existe tanto, si lo hubiera, y hubiera algún caso, sí que es verdad que se daría este tipo de control social por los tipos de relación, pero yo me decanto porque no existe... en todo caso es un poco la presión social te lleva a hacer muchas cosas que tú por tú cuenta no harías. Por ejemplo, las mesas de trabajo que hacemos, yo creo que hay veces que la gente participa porque como lleva participando tantos años, para no desvincularse..." (E9)

Aunque no hay una referencia a normas o reglas no escritas que sirvan como sanciones en el caso de actuaciones inaceptables en el sector, sí que existe un reforzamiento de la colectividad hacia las cosas que "deben hacerse". Tanto en entrevistas como en grupos de discusión se enfatizó en la colaboración en zonas próximas y cómo a partir de esas actividades conjuntas se genera una mayor cohesión con las organizaciones involucradas

"...Se ha avanzado en los últimos años, desde mi punto de vista... El hacer cosas conjuntas sí que ha hecho que tengamos un sentimiento de pertenencia. [Esas actividades son] como el pegamento, también es verdad que esto depende mucho de la persona que te coordine..."

Las entrevistas y grupos de discusión ofrecieron datos complementarios a las encuestas. En ambas técnicas se coincidió en que hay mayor relación con aquellas que comparten temas similares. También es posible que las pequeñas se identifiquen más con las pequeñas o con alguna que sea influyente. Pero sobre todo, el aspecto de los recursos humanos de dichas organizaciones constituye un elemento diferenciante. Se relacionan porque tienen vínculos en otros ámbitos. Destacan como ventajas del sector que las personas se conocen entre sí, hay un contacto personal y se trabaja a nivel de relación personal ya que es "más fácil cuando se conoce a alguien". Depende mucho de las personas, de los intereses de las personas que están en la entidad y de la entidad misma. En ese sentido, en contraste con la dispersión geográfica de su población y por ende de sus organizaciones, Teruel es pequeño

"...somos los mismos en las mismas cosas. Nos encontramos siempre..."

Al preguntar si percibían su red como una estructura abierto o cerrada, se decantaban discursivamente por la primera opción. El objetivo es ser una estructura abierta, "otra cosa es que se consiga o no". Ha habido muchas personas que se han salido de algunas entidades por el problema de que son casi unipersonales, o bi-personales o tri-personales y ha habido un cansancio. Quizás en este sentido, la intermediación personal juegue un papel importante a favor y en contra. Y es que como decía una de las especialistas entrevistadas 
"...depende de las relaciones que se establezcan. Aquí en Teruel tenemos la peculiaridad de que somos los mismos prácticamente para todo, y entonces estamos como más en familia... pero si acabamos siendo siempre los mismos, aunque no por ello es cerrado. Están abiertos a otras posibilidades..." (E5)

Una explicación para la redundancia de los miembros del TSAS es que las entidades profesionalizadas tienden a tener plantillas bastante estables, en tanto que la persona que es voluntaria, suele estar en varias organizaciones. Sin embargo, sí hubo mención explícita a que hay aspectos negativos en esa configuración

"...al ser más pequeño no se renueva la gente, es más fácil estancarse. Es un punto negativo porque es un círculo cerrado y es un punto positivo porque se favorece la comunicación y la confianza..." (E6)

\section{9.- Motivos para establecer relaciones con otras entidades}

En relación a la pregunta sobre los motivos que llevan a establecer relaciones con otras entidades, de las 49 entidades encuestadas, un $72,7 \%$ de ellas indica que el motivo principal es la persecución de los mismos intereses. Es importante destacar que, como refleja la Tabla 3, la proximidad geográfica sea poco relevante para establecer relaciones tal y como se había pensado en un inicio. Sin embargo, ese factor, junto con la confianza y las estrategias para obtener apoyos económicos pueden ser un área de oportunidad para establecer vínculos con otras entidades del TSAS en la provincia de Teruel.

\section{Tabla 3. Motivos para establecer relaciones con otras entidades}

\begin{tabular}{|lc|}
\hline Mismos intereses & $72,7 \%$ \\
Proximidad geográfica & $11,4 \%$ \\
Confianza & $11,4 \%$ \\
Es una condición para obtener apoyos económicos & $2,3 \%$ \\
Otro & $2,3 \%$ \\
\hline
\end{tabular}

FUENTE: Elaboración Propia. 
Si como establece Uzzi (1997), y algunos trabajos posteriores como los de Vota y otros (2012), las relaciones arraigadas se fundamentan en la confianza, la transferencia de información estratégica y los acuerdos conjuntos para la resolución de problemas, podemos inferir que las relaciones entre las asociaciones del TSAS son arraigadas, principalmente las que se congregan en macro-agrupaciones y plataformas. Esta afirmación se sustenta en la disposición a colaborar y participar en organizaciones de segundo nivel que requieren un esfuerzo para llegar a acuerdos y un proceso previo que implica frecuencia e intensidad en las interacciones.

\section{0.- Relaciones con instituciones}

En la tabla 4 se muestran las respuestas al punto de la encuesta en que se pregunta con qué instituciones se tiene una relación establecida. Hay una predominancia manifiesta hacia instituciones oficiales. El 70,5\% de las entidades expresa que la institución con la que más se relacionan es el Ayuntamiento, seguido del Gobierno de Aragón. En menor grado, con los espacios universitarios locales y sus fundaciones. La tabla 4 indica la proporción de los resultados.

\section{Tabla 4. Relaciones con las instituciones}

\begin{tabular}{|lccc|}
\hline & Siempre & A veces & Nunca \\
\hline Ayuntamiento & $70,5 \%$ & $25,0 \%$ & $4,5 \%$ \\
Gobierno de Aragón & $61,4 \%$ & $29,5 \%$ & $4,5 \%$ \\
Diputación Provincial de Teruel & $56,8 \%$ & $25,0 \%$ & $13,6 \%$ \\
Comarca & $54,5 \%$ & $27,3 \%$ & $11,4 \%$ \\
Instituto Aragonés de Servicios & $40,9 \%$ & $36,4 \%$ & $13,6 \%$ \\
Entidades representativas & $36,4 \%$ & $22,7 \%$ & $31,8 \%$ \\
Universidad de Zaragoza & $18,2 \%$ & $29,5 \%$ & $40,9 \%$ \\
Obras sociales de cajas de ahorros & $15,9 \%$ & $59,1 \%$ & $18,2 \%$ \\
Iglesia & $13,6 \%$ & $15,9 \%$ & $56,8 \%$ \\
Universidad de Zaragoza - Campus de Teruel & $13,6 \%$ & $36,4 \%$ & $36,4 \%$ \\
Fundación universitaria Antonio Gargallo & $0,0 \%$ & $13,6 \%$ & $70,5 \%$ \\
\hline
\end{tabular}

FUENTE: Elaboración Propia.

Estos resultados son coincidentes con la forma de establecer vínculos para obtener recursos: un $61,4 \%$ de las entidades encuestadas considera que establece vínculos prioritariamente con las entidades de la provincia de Teruel, mientras que un 11,3\% establece vínculos con entidades externas a la provincia. 
Con referencia a la relación con dichas instituciones, destaca que el 95,4\% de los encuestados manifiestan que las relaciones son muy importantes para mejorar los servicios a los usuarios. El 61,4\% de las respuestas manifestó que su organización se beneficia de la información que ellas difunden en contraste con sólo un 6,8\% que muestra su desacuerdo sobre esta afirmación. Asimismo, un 56,9\% indica que la relación con dichas instituciones les ha ayudado a establecer contacto con otras entidades, mientras que un $15,9 \%$ de las organizaciones encuestadas se muestra en desacuerdo sobre esa afirmación.

La mayoría (59\%) está de acuerdo con que el grado de redundancia de la información que se intercambia con las instituciones es muy alto, por lo cual es concluyente que las instituciones mencionadas tienen un papel estratégico en la distribución de información entre las entidades del tercer sector. En este sentido es importante referir que un $43,1 \%$ considera que las entidades y las instituciones tienen un alto de grado de conocimiento mutuo, en contraste con un $20,4 \%$ de las entidades encuestadas que señalan la existencia de un bajo nivel de conocimiento.

Por otro lado, en la cuestiones que se refieren a la existencia de objetivos compartidos o comunes entre entidades externas a la organización pero localizadas en la misma provincia o en la misma comarca, un $75 \%$ de las entidades indican que se comparten objetivos con entidades de la misma provincia, y un $61,4 \%$ señalan la existencia de objetivos comunes en la misma comarca. Por lo que se puede concluir que las entidades encuestadas consideran que sí se comparten objetivos comunes. Tras realizar diversas pruebas, como fue el grado de centralidad, se constató que el Ayuntamiento de Teruel juega un papel importante en las relaciones con las entidades, es decir, es el que más se relaciona. Por el contrario, la institución que menos se relaciona con las entidades es la Fundación Universitaria Antonio Gargallo. Se trata de una red muy densa en la que todos sus nodos están, en mayor o menos medida, relacionados. En este caso, no existe ningún nodo desconectado de la red. La densidad, que indica la proporción de relaciones que se dan respecto al total de posibilidades, es del 10,23\%. Dado el alto número de nodos existentes, este resultado indica una densidad relevante en la red.

\section{1.- Flujos de información en las redes del TSAS turolense}

En términos de manejo de información hay un contraste de opiniones. Hay una cierta transferencia de información que beneficia a todas las entidades que permite incrementar las opciones de conducta y hacer una predicción de las necesidades a largo plazo o nuevas oportunidades que se puedan plantear; sin embargo no existe una percepción consensuada. En la parte cualitativa del estudio se obtuvieron visiones encontradas como aquella que considera que "no se transmite. Cada uno quiere 
su parcela". Mientras otra afirma que "sí existe, pero podríamos hacer más." La representante de una organización pequeña afirmaba "Tienes que estar pendiente del BOE, si no tienes personal, se te pasan las oportunidades. Nadie te avisa". La percepción de que la información depende de la relación personal es generalizada y debe profundizarse con otro tipo de instrumentos en estudios posteriores. El siguiente testimonio ilustra el proceso de búsqueda de información en un miembro activo de la red

"... Lo de descolgar el teléfono es habitual, no hace falta llevar unos canales formales, un trabajador social en una jornada laboral puede hablar con dos o tres entidades al día fácilmente (con una administración y con dos entidades) para resolver cualquier tipo de incidencia, es fácil eso, eso es un trabajo en red. Ese trabajo en red que se hace de llamar de una entidad a otra permite a la organización mejorar el servicio, ya que muchas veces, si no se trabajara en red, muchas de las actividades que se realizan con usuarios no se podrían llevar a cabo.." (E3)

En un grupo de discusión se mencionaba

"La gente que va perdida, tiene la opción de preguntar, a mí siempre me han ayudado en todo lo que he preguntado. Pero se reflexionaba conjuntamente que quizás es por la relación personal. Nadie te avisa de nada. Se comparten mínimos recursos" (GD2)

Hay una conciencia de la necesidad de compartir más recursos. "La colaboración ayuda a resolver problemas e incrementar las capacidades" menciona una de las entrevistadas, pero durante las entrevistas una situación común era que al hablar de recursos compartidos se pensaba que se hablaba de infraestructuras o espacios físicos. Otro tipo de recursos que se destacaba también era el voluntariado "Nos prestamos al voluntariado. Quedamos muy contentos. Mi hijo es el informático" mencionó una de las representantes en uno de los grupos de discusión.

Con respecto a la transferencia de información estratégica y acuerdos conjuntos, destaca un intercambio de información, otra vez, mediante aquellas que están integradas en agrupaciones o plataformas, en contraste con las que están aisladas. Por lo que podríamos constatar que los actores "mejor conectados en su red poseen una ventaja competitiva sobre los pobremente conectados" (Marcuello et al., 2010).

"...Es muy diferente hablar de esta plataforma que estamos siempre juntos, que cuando ya hablas en general. Entre nuestro sector (cooperación al desarrollo) nos ayudamos más...." (E4)

"...nos conocemos o mejor dicho, las que formamos el punto de encuentro, sí..." (E6)

Contrasta esta situación con las organizaciones pequeñas que generalmente están más desconectadas. En las entrevistas destaca que las organizaciones pequeñas perciben menos confianza entre las organizaciones del TSAS. Sin embargo, entre algunas organizaciones, 
"...también se mantienen las distancias en las que las actividades no son compartidas, porque en las entidades sociales también hay una cierta competencia (pero una cierta competencia, no hay una competencia como tal)..." (E5)

Hay una diferenciación al referir la relevancia de la información que obtienen de la red de vínculos del TSAS. El 36,4\% de los encuestados manifiesta que obtienen mayor información de entidades 0 instituciones de fuera de la provincia, mientras que un porcentaje muy similar, el $25 \%$, opina que la información relevante para su entidad procede de personas o entidades del interior de la provincia. Esto depende también del uso de herramientas informáticas que tiende a llegar a los dos extremos. Como lo explicaba una de las especialistas locales

“...Hay entidades que están muy profesionalizadas entonces y por ende maneja aplicaciones informáticas, internet, correo electrónico... más o menos todo lo que existe. Y luego existe la pequeña asociación (generalmente de personas mayores) que las que al final acaban teniendo un alto volumen acaban contratando una trabajadora social, y es ella la que se encarga de todo. Depende porque la provincia está muy envejecida, y en muchas organizaciones los voluntarios tienen una media de edad alta, entonces no acceden al uso de nuevas tecnologías, aunque hay otros que sí, pero es una barrera..." (E8)

A este respecto, hay que señalar que el fenómeno de las nuevas tecnologías es indiscutible y que sus efectos han alcanzado todos los ámbitos de la sociedad (Llamosas, 2015), por lo que las entidades del Tercer Sector de Acción Social no pueden quedar al margen de ese fenómeno y desaprovechar sus ventajas.

\section{2.- Conclusiones}

Tomando como referencia el trabajo de Coleman (1988) y Uzzi (1997) sobre las redes cerradas que favorecen los fuertes vínculos y siguiendo la perspectiva de Rowley, Behrens y Krackhardt (2000) Dyer y Nobeoka (2000) y Ahuja, Soda \& Zaheer (2012), consideramos que se requiere mayor investigación empírica para conocer cómo las organizaciones en diferentes ámbitos se benefician de los vinculos sociales que construyen a partir de sus relaciones, y cómo utilizan dichos vínculos en estrategias colectivas. En este sentido, el caso de Teruel, con sus características geográficas y poblacionales, se configura como un estudio de caso extremo.

En este trabajo se ha abordado el estudio del Tercer Sector de Acción Social en la provincia de Teruel, una provincia que se caracteriza por unos índices muy bajos de población con una elevada 
tasa de envejecimiento y con baja capacidad de renovación, por lo que las entidades del TSAS tienen retos importantes para llevar a cabo sus objetivos de intervención social, lo que se hace necesario adoptar una estrategia que les permita ser eficaces en su organización interna como sector.

Para garantizar mejores condiciones en la prestación de bienes y servicios por parte del TSAS es necesario consolidarse como actor social, aumentar la incidencia política y social del Sector. Para ello es necesario conocer los problemas del TSAS. En el contexto turolense si bien existe relación articulada con la sociedad civil, particularmente en sus aspectos sectoriales, predomina una débil articulación entre lo territorial y lo estatal, que repercute tanto en la incidencia social como en la política. Se percibe una lejanía de las estructuras estatales contrastada con una gran cercanía a las organizaciones del sector con las que se comparten actividades en lo local.

Además de una intensificación de la competencia en la provisión de bienes y servicios que afectan la eficacia de la intervención del TSAS, evidenciada por la poca información que fluye en las estructuras, los resultados sugieren también una atomización de las organizaciones más pequeñas en contraste con una creciente especialización por parte de las organizaciones más grandes, producto de la profesionalización, circulación de personal voluntario, etc.

El análisis de redes resultó relevante para entender cómo fluye el conjunto de recursos arraigados dentro de su red de relaciones sociales (Nahapiet y Ghoshal, 1998). Se han examinado distintas características estructurales de la red de relaciones manifestadas por las entidades entrevistadas que tienen que ver con la forma de recabar información útil para su entidad, así como las características de las redes establecidas. Por otro lado, el análisis de los datos cualitativos a través de la técnica de análisis del discurso, ha permitido matizar las respuestas y profundizar en los rasgos sustantivos de las relaciones que establecen las entidades, en cuanto a la existencia de confianza y reciprocidad. El análisis de redes resultó limitado en algunas de sus visualizaciones en tanto que el entramado denso ofrecido por el paquete tecnológico no resultaba explicativo o significativo para un observador no especializado. Al respecto han sido más útiles las visualizaciones de redes habituales o esporádicas.

Hemos encontrado que la estructura de la red de entidades del TSAS de la provincia de Teruel tiene carácter centralizado, ya que tres entidades de tamaño medio constituyen los nodos de referencia para la cohesión de la red. Asimismo, la estructura de la red de relaciones entre el TSAS y el sector público es considerablemente más densa que la existente en el interior del Tercer Sector, lo que permite concluir que existe una estrecha relación entre el Primer y el Tercer Sector (basada en acuerdos de colaboración, acciones complementarias, financiación a través de conciertos y subvenciones, etc.), la gran dependencia financiera del TSAS respecto de estas instituciones y la centralidad del Ayuntamiento de Teruel como nodo central por el que pasa buena parte de la información de los distintos actores. 
La integración de una perspectiva metodológica mixta permitió explorar otros ángulos del sistema analizado. A través de las entrevistas y grupos de discusión se pudo conocer las percepciones de las interacciones de la red del TSAS, concluyendo que los actores mejor conectados en su red poseen una ventaja competitiva sobre los pobremente conectados y eso depende no sólo de sus competencias tecnológicas sino también de otros capitales relacionales y simbólicos.

En este sentido, las redes establecidas entre las organizaciones tienen una fuerte cohesión interna pero una débil conexión externa. El trabajo cualitativo ha permitido evidenciar y profundizar las razones de esta tendencia: el sentido latente que los actores confieren a su pertenencia y acción en estas redes tiene que ver con el fomento de la sociabilidad de sus miembros, especialmente la del voluntariado.

En cuanto el sentido de pertenencia e identidad, observamos que en lugar de una identidad explícita heterocentrada (que hace suya los valores y principios de la solidaridad y el altruismo), nos encontramos con un alto sentido de pertenencia organizacional y territorial que se traduce en una identidad implícita autocentrada por la cual los empleados y voluntarios del TSAS de la región se identifican con las organizaciones territoriales con más fuerza que con los valores y principios universales.

Del mismo modo, podemos concluir que existe una construcción colectiva del capital social pero a través del fortalecimiento de redes intrínsecas de vinculación intraterritorial, y no un capital social que conecte con redes extrínsecas con carácter interterritorial (en la Comunidad Autónoma o el Estado). En esta construcción de redes intrínsecas juega un importante papel la interacción cara a cara, la confianza interpersonal y las relaciones afectivas.

Las implicaciones de este trabajo pueden ser relevantes a nivel conceptual al proporcionar evidencia de un estudio de caso extremo y a nivel de conocimiento del sector del TSAS de la provincia de Teruel en tanto profundiza la existencia de debilidades en las relaciones entre entidades, facilita un mapa de las relaciones y pone de relieve las entidades mejor conectadas y por ende mejores socias para el resto de entidades. Se espera que los resultados de la investigación pueden aportar un diagnóstico del caso del TSAS en la provincia de Teruel que permita contribuir al fortalecimiento de un sector estratégico para la sociedad de ese territorio. Además, se propone como futuras líneas de trabajo el estudio de las relaciones del TSAS con otras organizaciones de la Economía Social de la provincia, como cooperativas 0 entidades financieras. En este sentido, conviene tener en cuenta que los bancos de crédito cooperativo debido a su arraigo territorial y espíritu mutualista, pueden contribuir de forma activa a la construcción, acumulación y difusión del capital social (Cermelli, 2015). 


\section{Bibliografía}

ADLER, P.S. \& KWON, S. (2002): "Social capital: Prospects for a new concept", Academy of Management Review, 27, 17-40.

AHUJA, G., SODA, G. \& ZAHEER, A. (2012): "The genesis and dynamics of organizational networks", Organization Science, 23 (2), 434-448.

ALDASHEV, G. MARINI, M. \& VERDIER, T. (2015): "Governance of non-profit and non-governmental organizations -within- and between- organization analyses: an introduction", Annals of Public and Cooperative Economics, 86, 1-5.

ALMAGUER KALIXTO, P., AMOZURRUTIA, J.A. \& MARCUELLO-SERVÓS, C. (2014): "Policy Processes as Complex Systems : The case of Mesoamerican Sustainable Development Initiative", Journal of Sociocybernetics, 12, 1/2, 31-52.

ANDERSON, A. \& JACK, S. (2002): "The articulation of social capital in entrepreneurial networks: A glue or a lubricant?", Entrepreneurship and Regional Development, 14, 193-210.

ARIÑO, A. (2008): "Articulación del Tercer Sector en España", Revista Española del Tercer Sector, $10,107-132$.

ARIZA, A., FERNÁNDEZ, V. \& TIRADO, P. (2016): "La incidencia de los valores culturales en el voluntariado: el caso de Europa", REVESCO, Revista de Estudios Cooperativos, 120, 7-34.

BASSI, A. \& VICENTI, G. (2015): "Toward a New Metrics for the Evaluation of the Social Added Value of Social Enterprises", CIRIEC-España, Revista de Economía Pública, Social y Cooperativa, 83, 9-42.

BAXTER, J. \& EYLES, J. (1997): "Evaluating qualitative research in social Geography: Establishing "Rigour" in Interview Analysis", Transactions of the Institute of British Geographers, 22(4), 505525.

BEER, S. (1977): Diseñando la Libertad, Col. BREVIARIOS, Fondo de Cultura Económica, México Madrid - Buenos Aires.

BOURDIEU, P. (1980): "Le capital social. Notes provisories", Actes de la Recherche en Sciences Sociales, 3, 41-56.

BOURDIEU, P. (1985): "The forms of capital". In: Richardson, J.G. (Ed.), Handbook of Theory and Research for the Sociology of Education, New York: Greenwood, 241-258.

BREHM, J. \& RAHN, W. (1997): "Individual-Level Evidence for the Causes and Consequences of Social Capital", American Journal of Political Science, 41, 999-1023. 
BUCIEGA, A. \& ESPARCIA, J. (2013): "Desarrollo, Territorio y Capital Social. Un análisis a partir de dinámicas relacionales en el desarrollo rural", Redes: revista hispana para el análisis de redes sociales, 24, 81-113.

BURT, R.S. (1992): "Social Structure of Competition". In: Nohria, N. \& Eccles, R.G. (Eds.), Networks and Organizations: Structure, Form and Action, Harvard Business School Press, Boston, MA.

CAMAGNI, R. (2003): "Incertidumbre, capital social y desarrollo local: enseñanzas para una gobernabilidad sostenible del territorio", Investigaciones Regionales, 2, 31-58.

CAREY, S., LAWSON, B. \& KRAUSE, D.R. (2011): "Social capital configuration, legal bonds and performance in buyer-supplier relationships", Journal of Operations Management, 29, 277-288.

CASANUEVA, C. \& GALÁN, J.L. (2002): "El análisis de redes sociales a partir de datos relacionales: aplicación al estudio del caso del sistema productivo local de Valverde del camino", VII Taller de Metodología y Técnicas de Investigación en Economía y Dirección de ACEDE, Benicassim.

CERMELLI, M. (2015): "Bancos de crédito cooperativo y crecimiento territorial, génesis de un modelo de desarrollo local y análisis del caso del Banco de Crédito Cooperativo 'G. Toniolo' de San Cataldo", Boletín de la Asociación Internacional de Derecho Cooperativo = Journal International Association of Cooperative Law, 49, 181-193.

CHANG, H.H. \& CHUANG, S.S. (2011): "Social capital and individual motivations on knowledge sharing: Participant involvement as a moderator", Information \& management, 48(1), 9-18.

COLEMAN, J.S. (1988): "Social capital in the creation of human capital", American Journal of Sociology 94, 95-120.

COLEMAN, J.S. (1990): Foundation of Social Theory, Harvard University Press, Cambridge, MA.

COLLANTES, F. \& PINILLA, V. (2003): "La evolución a largo plazo de la población española, 18602000: Tipología provincial y análisis del caso aragonés". En: Pinilla Navarro, V. \& Sáez Pérez, L. A. (coords). Políticas demográficas y de población, Gobierno de Aragón, Zaragoza, 41-70.

CUÑAT, R. (2015): "Las redes como factor clave para la consolidación de nuevas cooperativas de trabajo asociado", REVESCO, Revista de Estudios Cooperativos, 119, 26-52.

DAVID, M.V. \& TACORONTE, M.I. (2015): "Ejes teórico-conceptuales del capital social en ámbitos rurales", Psicogente, 17(31), 49-66.

DE LA GARZA, E., LÓPEZ, E.V. \& ROJAS, E.C. (2012). "La confianza como elemento del capital social en una pequeña empresa de la industria metalmecánica de la región centro de Coahuila, México", Global Conference on Business and finance Proceedings, 7 (2), 1471-1476.

DEBERTIN, D.L. \& GOETZ, S.J. (2015): Social Capital and Economic Growth: A New model for Rural Communities, University of Kentucky, Department of Agricultural Economics, No. 202763.

DYER, J.H. \& NOBEOKA, K. (2000): "Creating and managing a high performance knowledge sharing network: The case of Toyota", Strategic Management Journal, Special Issue 21, 345-67. 
FISCHER, F. (2003): Reframing public Policy. Discursive Politics and Deliberative Practices, Oxford University Press, Oxford.

GARCÍA BOUTIGUE, R. (2006): Sistemas Complejos. Conceptos, método y fundamentación epistemológica de la investigación interdisciplinaria, Editorial Gedisa, Barcelona.

GRANOVETTER, M. (1973): "The strength of weak ties", American Journal of Sociology, 78, 13601380.

GRANOVETTER, M. (1985): "Economic Action and Social Structure: The problem of Embeddedness", American Journal of Sociology, 91, 481-510.

GULATI, R., NORHIA, N. \& ZAHEER, A. (2000): "Strategic networks", Strategic Management Journal, 21, 203-215.

HANNEMAN, R.A. (2001): "Introduction to Social Network Methods". Disponible en http://faculty.ucr.edu/ hanneman/nettext/

HITT, M.A., IRELAND, R.D., SIRMON, D.G. \& TRAHMS, C.A. (2011): "Strategic entrepreneurship: creating value for individuals, organizations, and society", The Academy of Management Perspectives, 25(2), 57-75.

HOLLENBECK, J.R. \& JAMIESON, B.B. (2015): "Human Capital, Social Capital, and Social Network Analysis: Implications for Strategic Human Resource Management", The Academy of Management Perspectives, 29(3), 370-385.

KOKA, B. \& PRESCOTT, J. (2002): "Strategic alliances as social capital: a multidimensional view", Strategic of Management Journal, 23, 795-816.

LI, Y. (2015): Handbook of Research Methods and Applications in Social Capital, Edward Elgar Publishing, Cheltenhan (UK).

LIN, N. (2001): Social capital: A theory of social structure and action, Cambridge University Press, New York.

LIPSET, S. \& TROW, M. (2004): "Union Democracy. The internal politics of the International Typographical Union". In: Yin, R., The Case Study Anthology, Thousand Oaks and Sage, London, UK, 113-124.

LLAMOSAS, A. (2015): "Las nuevas tecnologías en la empresa: especial referencia a las cooperativas", Boletín de la Asociación Internacional de Derecho Cooperativo = Journal International Association of Cooperative Law, 49, 307-320.

MALECKI, E. (1995): "Culture as mediator of global and local forces". In: Van der Knaap, B. \& Le Heron, R. (Eds.), Human resources and industrial spaces: A perspective on globalization and localization, John Wiley \& Sons, Chichester, 105-127. 
MARBÁN, V. \& RODRÍGUEZ CABRERO, G. (2008): "Panoramic view of the social third sector in Spain environment, development, social research and challenges", Revista Española del Tercer Sector, $9,13-39$

MARCUELLO, C., DÍAZ, M., BELLOSTAS, A., FILGUEIRA, M., GARCÍA, A., MARTíNEZ, M., MARCUELLO, CH., SAZ, I. \& SERRANO, C. (2010): Plataformas aragonesas: análisis desde el enfoque de la gobernanza y los procesos de participación. Disponible en http://aragonparticipa.aragon.es/sites/default/files/plataformas_aragonesas.pdf

McFADYEN, M. \& CANNELLA, A. (2004): "Social capital and knowledge creation: diminishing returns of the number and strength of exchange relationships, Academy of Management Journal, 47, 735746.

MOLINA, J. (2012): "Mixed Methods Research in Strategic Management: Impact and Applications", Organizational Research Methods, 15 (1), 33-56.

MORGAN, G. (1986): Images of organizations, Sage, Beverly Hills.

MULLEN, B. \& COOPER, C. (1994): "The relation between group cohesiveness and performance: An integration", Phychological Bulletin, 115, 210-277.

MUR, M. \& ARTERO, I. (1998): "Teruel. Población y economía. Últimas tendencias", Revista de relaciones laborales, 6, 161-184.

NAHAPIET, J. \& GHOSHAL, S. (1998): "Social capital, intellectual capital, and the organizational Advantage", Academy of Management Review, 23, 242-266.

OINAS, P. (1998): The Embedded Firm? Prelude for a revived geography of enterprise, Helsinki School of Economics and Business Administration, Helsinki.

PALDAM, M. (2000): "Social Capital: One or Many? Definition and Measurement", Journal of Economic Surveys, 14, 629-653.

PERALDI, X. \& ROMBALDI, M. (2009): "Contribution of the third sector to the rural development: Some comments in the Corsica context", Geographie Economie Societe, 11(2), 161-179.

PLATAFORMA DE ONG DE ACCIÓN SOCIAL (2006): Plan Estratégico del Tercer Sector de Acción Social, Madrid: Plataforma de ONG de Acción Social.

PLATAFORMA DE ONG DE ACCIÓN SOCIAL (2013): II Plan Estratégico del Tercer Sector de Acción Social, Madrid: Plataforma de ONG de Acción Social.

PLATAFORMA DEL VOLUNTARIADO DE ESPAÑA (2013): Diagnóstico del Tercer Sector de Acción Social, Madrid: Plataforma del Voluntariado de España.

PUTMAN, R.D. (1995): "Bowling alone: America's declining social capital", Journal of Democracy, 6, 65-78. 
PwC (2013): Estudio sobre el presente y futuro del Tercer Sector social en un entorno de crisis, PwC, Esade, La Caixa, Barcelona.

ROWLEY, T., BEHRENS, D. \& KRACKHARDT, D. (2000): "Redundant Governance Structures: An Analysis of Structural and Relational Embeddedness in the Steel and Semiconductor Industries", Strategic Management Journal, 21, 369-86.

RUBIO, P. (1996): "La crisis del mundo rural. Experiencias de desarrollo en la provincia de Teruel". Lección inaugural del curso académico 1996-97, Zaragoza: Secretariado de Publicaciones de la Universidad de Zaragoza.

SCHMITZ, H. (1995): "Small Shoemakers and Fordist Giants: Tales of Superclusters", World Development, 23, 9-28.

SMITH, G., MALONEY, W. \& STOKER, G. (2004): "Building social capital in city politics: scope and limitations at the inter-organisational level", Political Studies, 52, 508-530.

TSAI, W. \& GHOSHAL, S. (1998): "Social capital and value creation: the role of intrafirm networks", Academy of Management Journal, 41, 464-478.

UGALDE, N. \& BALBASTRE, F. (2013): "Investigación Cuantitativa e Investigación Cualitativa: Buscando las ventajas de las diferentes Metodologías", Ciencias Económicas, 31 (2), 179-188.

UZZI, B. (1997): "Social structure and competition in interfirm networks. The paradox of embeddeness", Administrative Science Quarterly, 42, 35-67.

VOTA, A., MARÍA, A., HERNÁNDEZ, O.A. \& LÓPEZ, J.C. (2012): "Redes y confianza: dimensiones del capital social en las microempresas rurales en Chihuahua, México", Nueva antropología, 25 (77), 31-57.

ZIMMER, A. \& FREISE, M. (2008): "Bringing society cack in: Civil society, social capital, and third sector". In: Maloney, W. \& Deth, J.v. (Ed.): Civil Society and Governance in Europe, Edward Elg, Cheltenham, 19-42. 